\title{
Spectrum Measurements supporting Reconfiguration in Heterogeneous Networks
}

\author{
T. Renk ${ }^{\dagger}$, C. Kloeck ${ }^{\dagger}$, F. K. Jondral ${ }^{\dagger}$, P. Cordier ${ }^{\diamond}$, O. Holland ${ }^{\triangleleft}$, and F. Negredo ${ }^{\star}$ \\ ${ }^{\dagger}$ Universität Karlsruhe (TH), Germany, \{renk, kloeck, fj\}@int.uni-karlsruhe.de \\ $\diamond$ France Telecom Research \& Development, France, pascal.cordier@francetelecom.com \\ $\triangle^{\triangle}$ King’s College London, United Kingdom, oliver.holland@kcl.ac.uk \\ * Telefonica I+D, Spain, fnd20@tid.es
}

\begin{abstract}
This paper represents spectrum occupation measurements performed during the Soccer World Cup 2006 in Germany. The measurements cover the range from $400 \mathrm{MHz}$ up to $2600 \mathrm{MHz}$ including among other communication standards GSM 900, DCS 1800, UMTS, and WiFi. The intention of the paper is to compare spectrum occupation during a mega-event to spectrum allocation in a "normal usage" situation and to investigate correlation between the received power levels and specific events, e.g., scoring within a match. These results form the basis for a metric that may be assigned for dynamic spectrum access in heterogeneous radio environments. Measurement results are deeply investigated with respect to time-dependent and peak power statistics as well as quality of service considerations. Quality of service in that case is represented in terms of succeeded voice calls, data calls and data transfers as well as average throughput.
\end{abstract}

Keywords-Spectrum Occupation Measurements, Spectrum Holes, Reconfiguration Management.

\section{INTRODUCTION}

$\mathbf{T}$ HERE is no doubt that the demand for bandwidth is increasing enormously in the near future, most notably with respect to innovative multi-media applications that tend to provide services whenever and wherever users ask for them [1], [2]. This ubiquitous scenario obviously requires new communication techniques that are able to overcome the inherent limitations of the radio frequency spectrum [3]. Such an approach is dynamic spectrum allocation [4] - [6]. Here, a radio device will not only be assigned to a specific communications standard, but will be able to use different standards with respect to its current requirements. This heterogeneous environment where a multitude of standards is coexisting comes along with suitable, reconfigurable radio concepts, so-called cognitive radios [7], [8].

In order to perform dynamic spectrum allocation in a heterogeneous network, proper knowledge about spectrum occupation is indispensable. The Soccer World Cup 2006 in Germany offered a great opportunity to obtain measurement data on spectrum allocation during a mega-event. For that purpose, measurements at two different sites have been performed, namely Kaiserlautern (Italy vs. USA) and Dortmund (Germany vs. Poland). To be able to compare the results during the matches to results at a "nor- mal day" occupation, measurements the day prior to the matches have been collected as well. The power level measurements ranged from $400 \mathrm{MHz}$ to $2600 \mathrm{MHz}$ including, e.g., GSM 900 downlink, DCS 1800 downlink, and UMTS downlink. Additionally, measurements with trace mobiles were made to evaluate the quality of connections.

The remainder of the paper is organized as follows. In Section II the measurement setup will be described. We give a brief survey of the measurement equipment and sites and the configuration of the measurement devices. Section III deals with measurement results that include timedependent statistics, complementary cumulative distribution functions, and analysis on trace mobiles. Finally, Section IV concludes the paper.

\section{Measurement SetuP}

\section{A. Measurement Equipment}

The measurements were performed on the basis of different measurement devices including spectrum analyzer and cellular dedicated trace mobiles (for GSM and UMTS). The spectrum analyzer was connected to an omnidirectional antenna placed at $4 \mathrm{~m}$ height above ground. It was controlled by the EM Spy Software developed by France Telecom (see Figure 1) for analyzer monitoring and data collecting and takes spectrum snapshots of frequency bands of interest periodically (about 1 second order of magnitude). Memory size of the analyzer and consequent tradeoffs between time and frequency resolution allowed automatic recording periods of about 30 to 45 minutes without manual intervention. Several cycles were therefore programmed to cover the whole duration of a soccer match.

Apart from these power level recordings further information on the network behavior was simultaneously obtained by trace mobiles. Communications were therefore triggered periodically for phone call and data transfer type of services. These devices were directly connected to a notebook and controlled via specific software.

\section{B. Measurement Sites}

The measurements were performed in fixed locations with equipment that was located in 2 dedicated vans. Given 


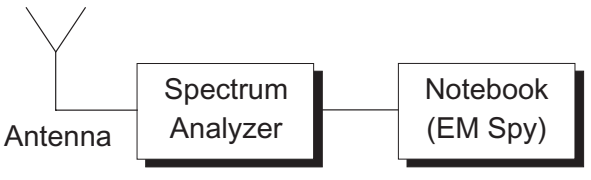

Fig. 1. Schematic description of spectrum analyzer with associated monitoring and recording software and omnidirectional antenna (height: $4 \mathrm{~m}$ ).

the importance of the event one major challenge consisted in finding adequate locations that were likely to give relevant information on wireless activity, taking security constraints and consequent security zones around the stadiums into account. Another important constraint was to keep the same locations the day before and during the events to allow proper comparison of measured data.

In Dortmund the vans were located in-between city center and stadium on one of the red marked paths to the stadium, hence providing a great opportunity to exploit "moving spots" due to pedestrians. The situation was different in Kaiserslautern where the vans were located in a parking lot approximately 400 meters away from the stadium entrance and not more than 30 meters from a path.

\section{Measurement Configuration}

Four different measurement configurations have been used that will be described in detail in the following.

1) GSM 900: Measurements were taken from $915 \mathrm{MHz}$ to $995 \mathrm{MHz}$, which covers the GSM 900 downlink band (925 MHz to $960 \mathrm{MHz}$ ). Frequency resolution was set to $200 \mathrm{kHz}$, thus one measuring point represents one channel in GSM. One spectrum snapshot was taken every second.

2) DCS 1800: Measurements were performed in a range from $1805 \mathrm{MHz}$ to $1885 \mathrm{MHz}$, which covers the DCS 1800 downlink band (1805 $\mathrm{MHz}$ to $1880 \mathrm{MHz})$. Frequency resolution was set to $200 \mathrm{kHz}$ as well, and one spectrum snapshot was taken per second.

3) UMTS downlink and WiFi: A frequency resolution of $1 \mathrm{MHz}$ was selected in a range from $2 \mathrm{GHz}$ to $2.6 \mathrm{GHz}$, which covers the UMTS downlink band (2110 $\mathrm{MHz}$ to $2170 \mathrm{MHz}$ ) and the WiFi band (2400 MHz to $2500 \mathrm{MHz}$ ). One spectrum snapshot per second was taken.

4) Global band: The global band covers the PMR bands (450 MHz to $470 \mathrm{MHz}$ ), the DVB-T bands (470 MHz to $860 \mathrm{MHz}$ ), and the bands from GSM 900 up to WiFi. Frequency resolution was set to $2 \mathrm{MHz}$, and a spectrum snapshot was collected every 4 seconds.

\section{Measurement Results}

\section{A. Time-dependent Statistics and Autocorrelation}

In this section we discuss some of the results from the spectrum measurement campaign. We concentrate on measurements from the $900 \mathrm{MHz}$ band in Dortmund and the $1800 \mathrm{MHz}$ band in Kaiserslautern, as these have proven

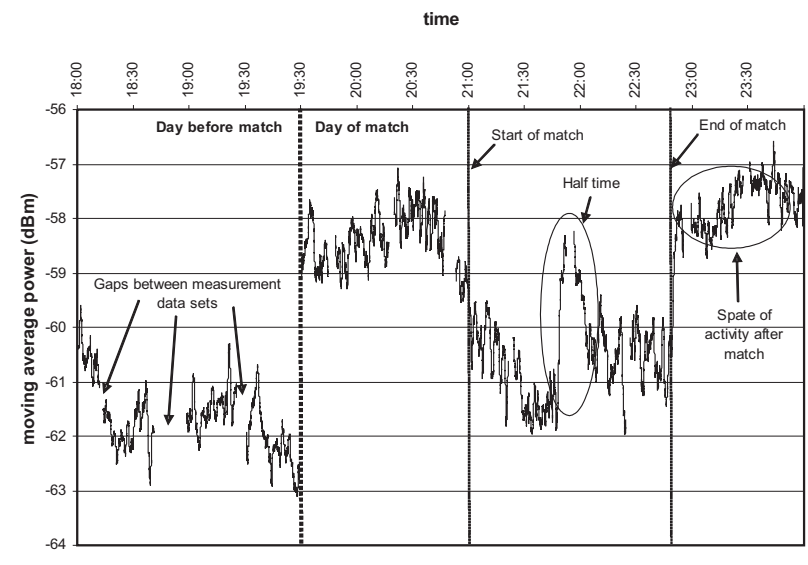

Fig. 2. Time-variation of the average observed power per $200 \mathrm{kHz}$ subband in the $900 \mathrm{MHz}$ band in Dortmund.

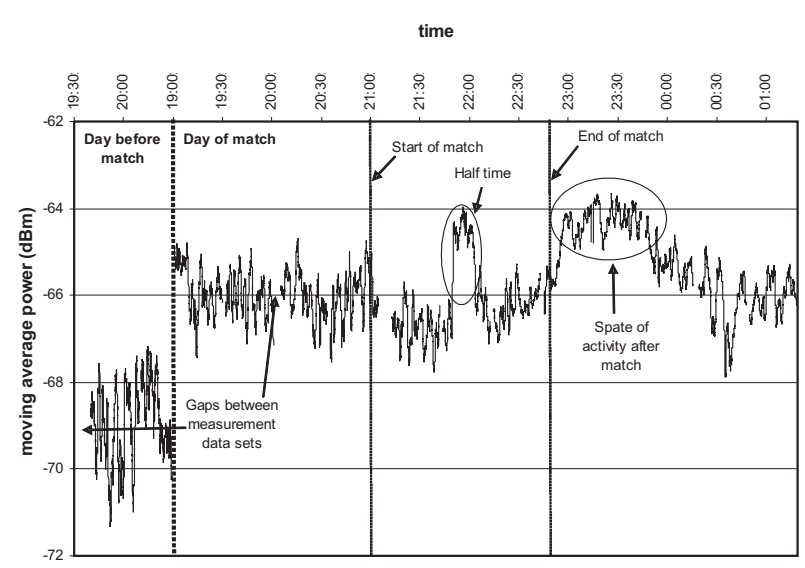

Fig. 3. Time-variation of the average observed power per $200 \mathrm{kHz}$ subband in the $1800 \mathrm{MHz}$ band in Kaiserslautern.

to be most interesting. Figure 2 plots a moving average (of order 60) of the average power level per $200 \mathrm{kHz}$ subband in Dortmund, while also denoting the exact timings of events concerning the match. Figure 3 is the associated plot for the $1800 \mathrm{MHz}$ band in Kaiserslautern. Moreover, the power-level statistics for different times with respect to the matches, both in Dortmund and Kaiserslautern, are in Table I.

From these plots, the relationship between power level and the timing of the match can clearly be seen. In both Dortmund and Kaiserslautern, the observed spectrum power on the day before the match is significantly lower than on the day of the match, by approximately $3 \mathrm{~dB}$. On the day of the match there is a high power as the build-up to the event proceeds. During the match (both in the first and second halves), the power level reduces significantly, presumably because people are engrossed in the match. This reduction for both Dortmund and Kaiserslautern is approximately $2-3 \mathrm{~dB}$. Notably, at half time there is a considerable peak in spectrum activity, and there is another particularly strong peak after the match. We speculate that the peak after the match is caused by (1) spectators leav- 
TABLE I

TIME-DEPENDENT STATISTICS FOR DORTMUND AND KAISERSLAUTERN

\begin{tabular}{lcccc} 
& \multicolumn{2}{c}{ Average Power $[\mathbf{d B m}]$} & \multicolumn{2}{c}{ Power Coefficient of Variation } \\
\hline & $\begin{array}{c}900 \mathrm{MHz} \\
\text { Dortmund }\end{array}$ & $\begin{array}{c}1800 \mathrm{MHz} \\
\text { Kaiserslautern }\end{array}$ & $\begin{array}{c}900 \mathrm{MHz} \\
\text { Dortmund }\end{array}$ & $\begin{array}{c}1800 \mathrm{MHz} \\
\text { Kaiserslautern }\end{array}$ \\
\hline Day before match & -61.6 & -68.8 & 0.258 & 0.493 \\
Build-up to match & -58.4 & -65.9 & 0.339 & 0.276 \\
First Half & -60.8 & -66.7 & 0.292 & 0.248 \\
Half Time & -59.2 & -64.9 & 0.299 & 0.260 \\
Second Half & -60.4 & -66.1 & 0.283 & 0.225 \\
Hour after match & -57.7 & -64.4 & 0.246 & 0.182
\end{tabular}

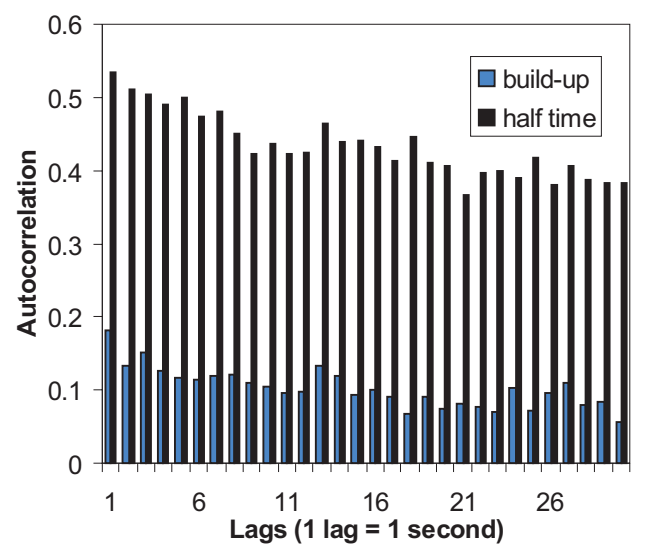

Fig. 4. Autocorrelation vs. time lags plots in the build-up to the match and at half time in Dortmund.

ing the ground and making calls as they move on to their next destination, and (2) perhaps calls being made to discuss the match, this latter cause might also be the case at half time. Note that there is a strong likelihood that particular events in the match influence the power level, e.g., the sending off of Pablo Mastroeni for the USA just before half time in Kaiserslautern, and the winning goal of Oliver Neuville for Germany just before full time in Dortmund. The "power surges" following these events are perhaps higher than would otherwise be expected. The trends observed here with respect to the timing of the match, possibly as well as events during the match, can be easily used in systems to dynamically allocate resources based on predicted variations in loads. Moreover, statistics such as the power coefficient of variation, also denoted in Table I, give a rough indication of the confidence with which a dynamic allocation can be made.

Next we consider the time-dependence of power levels through investigating the autocorrelation structure as an indication of the rate of power variability: note that a heavier tail in an autocorrelation vs. time lags plot indicates a tendency for power levels to vary slowly, hence more time for the system to react to dynamically allocate resources. Here we point out some noticeable trends. On the day before the match, the autocorrelation decreases slowly, only to approximately 0.35 within 30 lags (30 seconds). During the

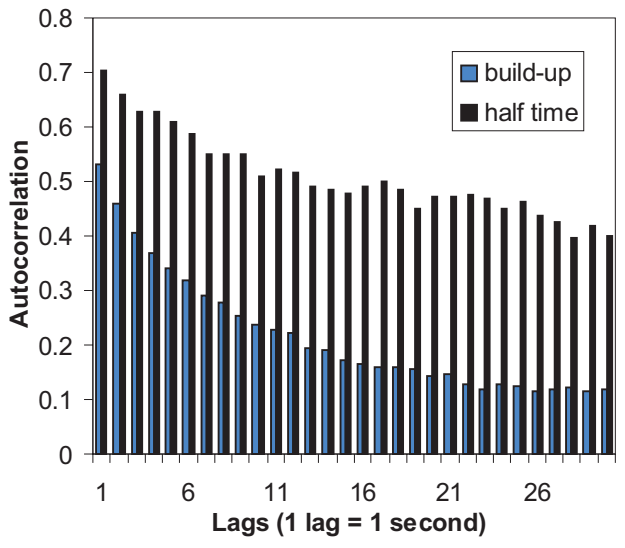

Fig. 5. Autocorrelation vs. time lags plots in the build-up to the match and at half time in Kaiserlslautern.

build-up to the match, the autocorrelation function drops extremely quickly, falling to approximately 0.1 within 30 lags thus indicating that the system must be rapidly reactive if it is going to be able to reallocate resources dynamically based on loads within this period. During the match itself, the autocorrelation function generally drops quite quickly. However, at half time it falls extremely slowly, only to approximately 0.4 within 30 lags. This represents a golden opportunity for the benefits of dynamic allocation to be leveraged, especially given the higher power levels observed at half time. Finally, in the hour after the match, the autocorrelation function again drops slowly, also representing a good opportunity for dynamic allocation. In general, it should be noted that there is a very good agreement between decays of autocorrelation plots for Dortmund and Kaiserslautern.

Figures 4 and 5 compare the autocorrelation plots for Dortmund and Kaiserslautern in the build-up to the match to those at half time, thus illustrating the enhanced potential for dynamic allocation, e.g., at half time. Note that in both cases the autocorrelation at lag zero is omitted for clarity.

\section{B. Complementary Cumulative Distribution Functions}

The complementary cumulative distribution function (CCDF) expresses the probability that a signal lies at or 


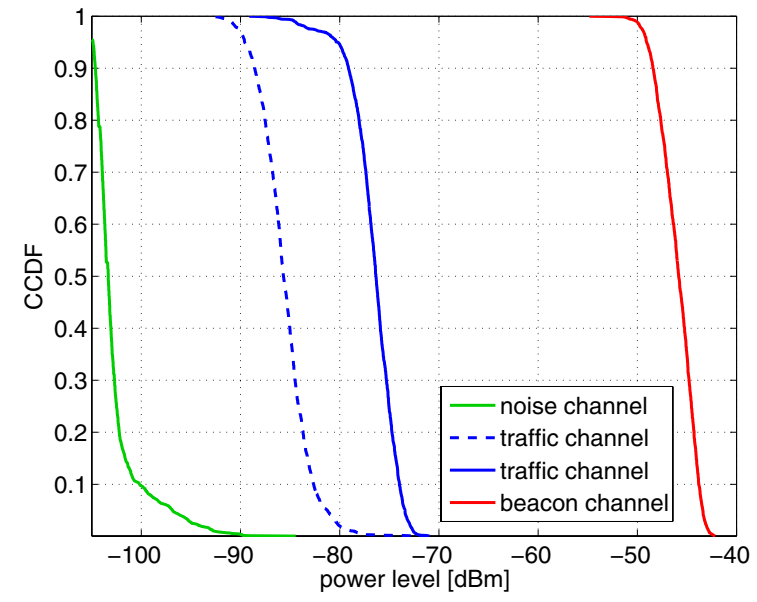

Fig. 6. Complementary cumulative distribution function of a noise channel, a traffic channel (dashed line indicates traffic the day before the match and solid line indicates the traffic on the day of the match), and a beacon channel in GSM 900.

above a specific power value. Thus, CCDF is a method to characterize the peak power statistics of digitally modulated signals.

Figure 6 depicts CCDF curves of a noise channel, a traffic channel, and a beacon channel of a GSM 900 system. A glance at the noise channel curve (green) shows that a detection threshold of $-90 \mathrm{dBm}$ can be chosen in order to decide whether a channel is free or currently allocated. This detection threshold depends on analyzer settings like resolution bandwidth, noise figure, and selected detector. It is obvious that this threshold serves as decision citerion for the detection of spectrum holes. The blue curves indicate measurements of a traffic channel the day before the match (dashed line) and on the day of the match (solid line). Not surprisingly, the power level on the day of the match is approximately 8 to $9 \mathrm{~dB}$ higher compared to those on the day before. This can definitely be ascribed to a higher density of used mobile phones. For instance, $95 \%$ of the power of the measured signal on the day of the match lie above $-80 \mathrm{dBm}$ and even $100 \%$ lie above the detection threshold of $-90 \mathrm{dBm}$, which indicates that this channel was used during the whole measurement period. The red line depicts the beacon channel that is occupied with a higher power level all the time (average power level is approximately $-46 \mathrm{dBm})$.

\section{Trace Mobiles}

Trace mobiles were used in order to perform quality of service measurements with respect to succeeded voice calls, data calls and data transfers as well as average throughput. In Dortmund, measurements in a $2 \mathrm{G}$ cellular system were collected whereas in Kaiserslautern we performed measurements in a $3 \mathrm{G}$ cellular network. Throughout this paragraph a succeeded event is denoted as OK and a failed event is denoted as NOK. Measurements have been set as follows. In Dortmund, a voice call lasted 62 seconds and FTP size was chosen to be 500 kbit. In Kaiserslautern,

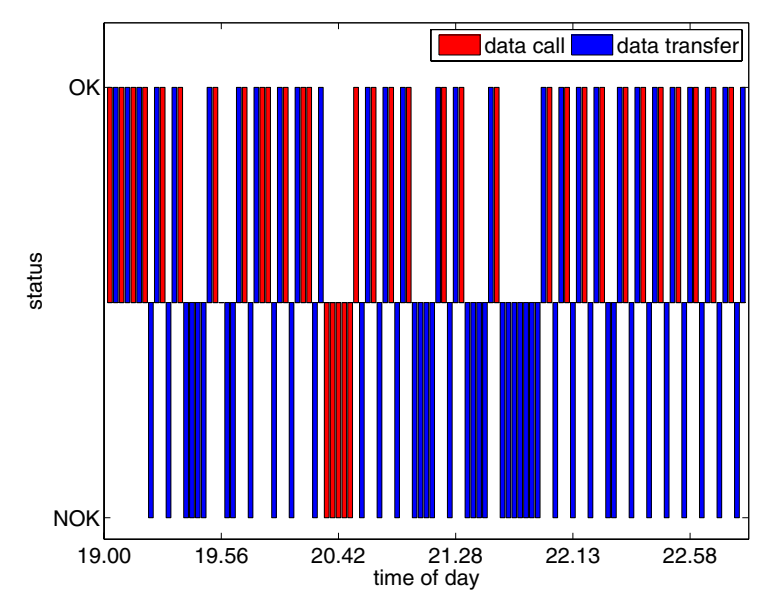

Fig. 7. Success $(\mathrm{OK})$ and failure $(\mathrm{NOK})$ of data calls and data tranfers in a $2 \mathrm{G}$ cellular system in Dortmund.

TABLE II

SUCCEEDED DATA CALLS AND DATA TRANSFERS FOR 2G

\begin{tabular}{lcc} 
& $\begin{array}{c}\text { Data calls } \\
\text { OK }[\boldsymbol{\%}]\end{array}$ & $\begin{array}{c}\text { Data transfers } \\
\text { OK }[\%]\end{array}$ \\
\hline Day before match & 100 & 100 \\
Build-up to match & 69 & 41 \\
First Half & 100 & 25 \\
Half Time & 100 & 50 \\
Second Half & 100 & 45 \\
Hour after match & 100 & 50
\end{tabular}

voice call duration was set to 60 seconds and FTP size was set to 3 Mbit. For the $3 \mathrm{G}$ measurements scrambling code 384 was used and no soft handover was considered. In both cases, voice calls and data calls were made alternatively. If a call failed, the procedure was repeated until a successful call could be made. Similarly, if a data transfer failed, the transfer was repeated until it succeeded.

In Dortmund, all calls were made over the same operator. As calls were made at a fixed location, they usually did not have any handover during the call. Only in the case of voice calls, some handovers could be recognized. All data calls started and ended in the same cell. Figure 7 shows succeeded and failed data calls and data transfers. It can especially be seen that from 20.40 to 20.45 five consecutive data calls failed. Additionally, during half time almost all data transfers failed (21.45 to 22.00). A precise percentage description is listed in Table II. The avergage throughput for a $2 \mathrm{G}$ cellular system is depicted in Figure 8 , where it can be seen that the average throughput suffers a tremendous degradation (approximately a factor of $1 / 5$ ) on the day of the match compared to the day before.

In Kaiserslautern, $100 \%$ of voice calls and $95 \%$ of data calls succeeded on the day of the match (measurements performed from 19.00 to 0.30 ). These facts in addition to the total number of calls are shown in Table III. In average, throughput is very good and there are only 4 failed data calls. It is reasonable to think that the majority of the 


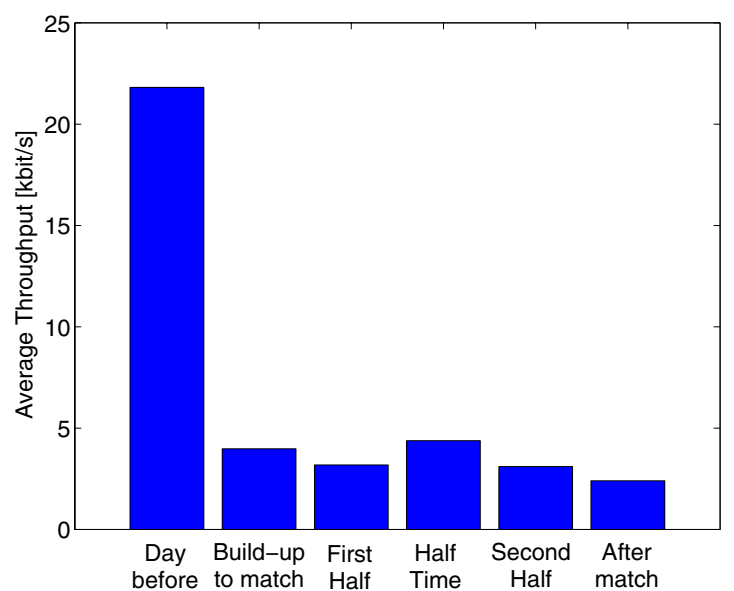

Fig. 8. Average throughput for a $2 \mathrm{G}$ cellular system in Dortmund.

TABLE III

SUCCEEDED DATA CALLS AND DATA TRANSFERS FOR 3G

\begin{tabular}{lcc} 
& Number of calls & OK [\%] \\
\hline Voice & 74 & 100 \\
Data & 74 & 95
\end{tabular}

calls were made in GSM, and for that reason the results are better in $3 \mathrm{G}$ than in $2 \mathrm{G}$. We consider four outstanding points of the curve plotted in Figure 9, however, the first two gaps cannot be assigned to any specific event: (1) data call failure shortly before the beginning of the match (20.55), (2) average throughput decrease in the time window from 21.22 to 21.27 most likely due to scoring (two goals were scored, first Italy scored in the 22nd minute and then USA scored in 27th minute), (3) data call failure during half time (21.45 to 22.00), and (4) data call failure shortly after the match (22.48). Decrease or even failure of calls can be related to congestion control. For that reason, when someone tries to make a call, the throughput is lower (spreading factor higher) since there are many users at the same time that try to call simultaneously.

\section{Conclusion}

In the near future, it will become necessary to create new techniques that are able to satisfy the increasing demand for more bandwidth. To achieve better system performance, reconfiguration in heterogeneous environments seems to be inescapable. In this paper results of spectrum measurements have been presented that serve as basis for the development of efficient and dynamic spectrum access algorithms. First, time-dependence of power levels has been investigated and autocorrelation functions as an indication of the rate of power variability have been calculated, where it can be seen that, e.g., half time during a match offers a great opportunity for dynamic allocation. Complementary cumulative distribution function have been calculated to characterize the peak power statistics of the received signals. Dependent on measurement settings a suitable detection threshold can be set that serves as indica-

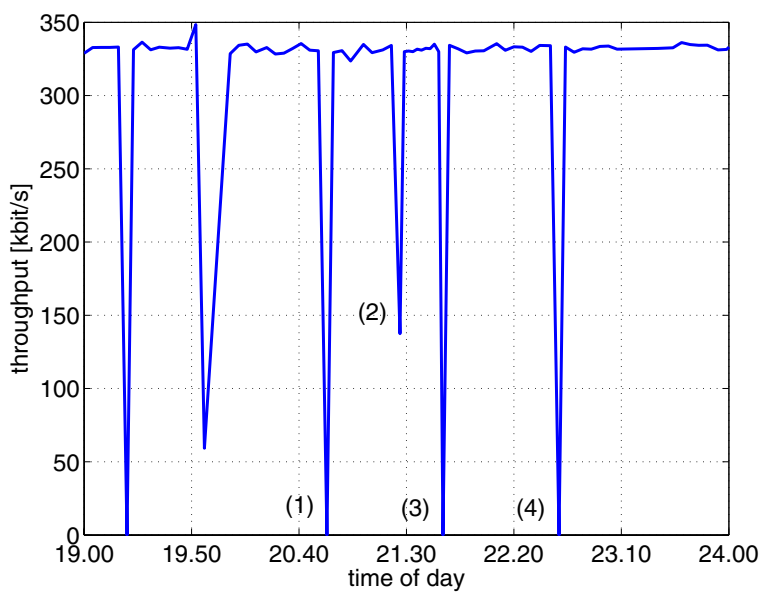

Fig. 9. Throughput for a $3 \mathrm{G}$ cellular system in Kaiserslautern.

tion for a free or occupied channel, a major point in the research area of cogntive radio. Finally, analysis of trace mobiles data showed that throughput highly depends on the communication standards ( $2 \mathrm{G}$ or $3 \mathrm{G}$ ) and, moreover, is correlated to specific events (start of the match, half time, scoring, etc.).

\section{ACKNOWLEDGMENT}

This work was performed in project $E^{2} R$ II which has received research funding from the Community's Sixth Framework program. This paper reflects only the authors' views and the Community is not liable for any use that may be made of the information contained therein. The contributions of colleagues from $\mathrm{E}^{2} \mathrm{R}$ II consortium are hereby acknowledged.

\section{REFERENCES}

[1] S. Verdu, "Wireless bandwidth in the making," IEEE Communications Magazine, pp. 53-58, July 2000.

[2] Q. Bi, G. I. Zysman, and H. Menkes, "Wireless mobile communications at the start of the 21st century," IEEE Communications Magazine, pp. 110-116, January 2001.

[3] Federal Communications Commission, "Spectrum policy task force report," ET Docket No. 02-135, November 2002.

[4] J. Zhao, H. Zheng, and G.-H. Yang, "Distributed coordination in dynamic spectrum allocation networks," First IEEE International Symposium on new Frontiers in Dynamic Spectrum Access Networks (DySPAN), Baltimore, Maryland USA, pp. 259-268, November 2005.

[5] P. Leaves, J. Huschke, and R. Tafazolli, "A summary of dynamic spectrum allocation results from DRiVE," IST Mobile and Wireless Telecommunications Summit, Thessaloniki, Greece, pp. 245-250, June 2002.

[6] D. Grandblaise, D. Bourse, K. Moessner, P. Leaves, "Dynamic spectrum allocation (DSA) and reconfigurability", SDR Forum, San Diego, California USA, November 2002.

[7] S. Haykin, "Cognitive Radio: Brain-empowered wireless communications," IEEE Journal on Selected Areas in Communications, vol. 23, no. 2, pp. 201-220, February 2005.

[8] J. Mitola III, "Cognitive Radio: An integrated agent architecture for software defined radio," Dissertation, Computer Communication System Laboratory, Department of Teleinformatics, Royal Institute of Technology (KTH), Stockholm, Sweden, 2000. 